\title{
Fast FEM/BEM Simulation of SAW Devices Via Asymptotic Waveform Evaluation
}

\author{
Vincent Laude, Member, IEEE, Alexandre Reinhardt, Mikaël Wilm, Abdelkrim Khelif, \\ and Sylvain Ballandras
}

\begin{abstract}
The finite element method/boundary element method (FEM/BEM) computation model applied to surface acoustic wave devices requires the solution of a large linear system for each frequency point. An asymptotic waveform evaluation technique is used to obtain an approximate solution of the linear system that is valid over a large frequency bandwidth. The approximate solution was shown to be very accurate and vastly reduces the computation time.
\end{abstract}

\section{INTRODUCTION}

$\mathrm{T}$ HE combination of the finite element method (FEM) and the boundary element method (BEM) for the simulation of surface acoustic wave (SAW) devices has been used by many authors [1]-[6]. Although the FEM/BEM method only applies to infinite periodic interdigital transducers (IDTs), and, thus, is only an approximation of a real finite SAW device, its high degree of accuracy and reasonable computational burden have made it the basis of most advanced SAW parameter extraction codes [3], [6][8]. Ventura et al. [8] have especially deeply optimized the FEM/BEM method by analytically integrating the asymptotic contributions of the spectral surface Green's function. It also has been used as a mathematical tool for the identification of the modes guided by the interface between a piezoelectric substrate and high aspect ratio metallic electrodes [9], and for the analysis of surface waves propagating obliquely in IDTs [10], [11].

The FEM/BEM method relies on an integral representation of the substrate surface assuming a semi-infinite medium through the spectral Green's function that relates the surface displacements to the surface stresses via a convolution operation, the BEM part, connected through a discrete formulation to the diffracting elements in the structure (i.e., the electrodes), which are treated acoustically by the FEM. The main output of the FEM/BEM is the harmonic admittance [12], a generalization of the strip admittance [13], [14]. The FEM/BEM is a spectral method. Although the spectral Green's function of a semiinfinite substrate does not depend on frequency, the FEM problem for the electrodes has to be solved for every frequency point. In practice, it turns out that this last computation becomes the main burden, especially if many frequency points are required as is almost always the case for

Manuscript received April 17, 2003; accepted October 19, 2003.

The authors are with the Laboratoire de Physique et de Métrologie des Oscillateurs/Centre National de la Recherche Scientifique (LPMO/CNRS), Besançon, France (e-mail: vincent.laude@lpmo.edu).

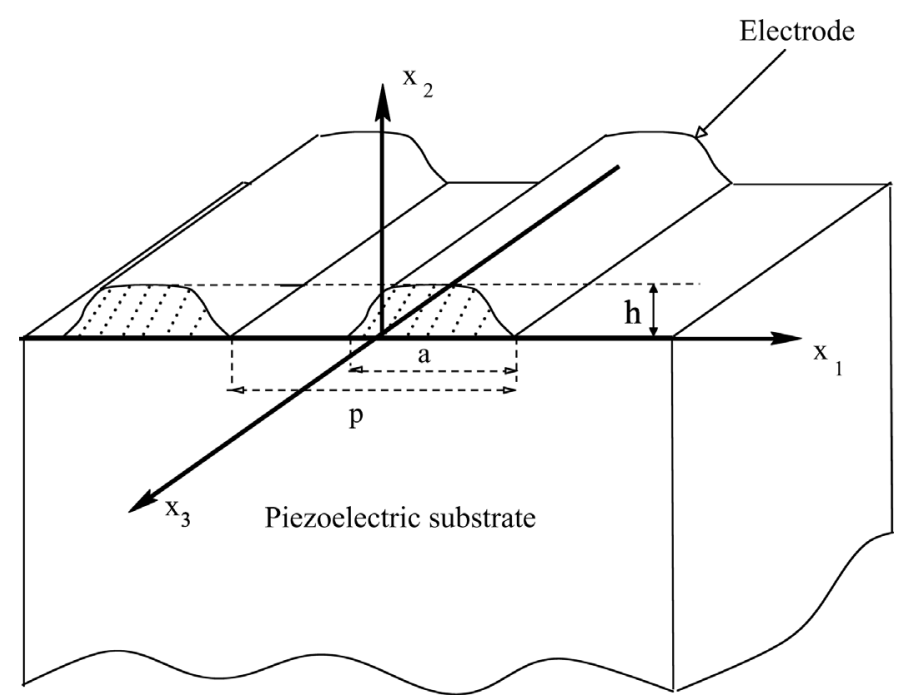

Fig. 1. Infinite periodic surface acoustic wave transducer.

the high quality-factor SAW resonators that are the basis of most SAW devices.

The purpose of this paper is to introduce an approximation method that requires the solution of the FEM problem only for the central frequency of the bandwidth of interest. This approximation method belongs to the larger class of asymptotic waveform evaluation (AWE) methods [15] [18], although it is here derived analytically from the particular form of the FEM problem. In particular, the ability of AWE methods to handle poles is not used. In Section II, the FEM/BEM method used is summarized briefly. The approximation method is derived in Section III. A computation example is given in Section IV, and the results are discussed. Section V is the conclusion.

\section{FEM/BEM METHOD}

The definitions of the axes and the considered geometry are given in Fig. 1. The structure is composed of a semiinfinite piezoelectric substrate extending in the half-space $x_{2}<0$, whose surface supports a periodic infinite metal strip grating. The grating electrodes are parallel to axis $x_{3}$, centered at $x_{1}=n p$, where $n$ is the electrode index and $p$ is the grating period. The width of the electrodes is denoted by $a$, so that $a / p$ is the metalization ratio. The electrodes are assumed to be long enough along $x_{3}$ that any dependence in $x_{3}$ can be omitted in the following equations. All considered fields are supposed to exhibit a time depen- 
dence $\exp (j w t)$, where $j$ is the imaginary constant and $\omega$ is the angular frequency.

An harmonic excitation is assumed to be applied to the infinite periodic grating, yielding the following form for the driving potential applied to the grating electrodes [12][14]:

$$
V_{n}(\gamma)=V_{0} \exp (-j 2 \pi n \gamma)
$$

where $\gamma$ is the characteristic parameter of the harmonic excitation. The alternating potential $+V,-V$ usually considered for a practical electrical excitation corresponds to $\gamma=$ $1 / 2$. For such an harmonic excitation, it has been shown that the electrode currents have the same dependence as their voltages [4] and that the ratio $Y=I_{n}(\gamma) / V_{n}(\gamma)$ does not depend on the actual number $n$ of the electrode. It is the so-called harmonic admittance (HA) [12].

The determination of the grating harmonic admittance has been the main goal of many theoretical and numerical works; we use in this paper the method due to Ventura et al. [4], [8]. Considering the harmonic excitation of (1), only one period of the infinite grating has to be considered in the analysis, and the convolution relation can be written:

$$
u_{i}\left(x_{1}\right)=\int_{-\frac{p}{2}}^{+\frac{p}{2}} G_{i j}^{(p)}\left(x_{1}-x^{\prime}\right) t_{j 2}\left(x^{\prime}\right) d x^{\prime},
$$

where $G_{i j}^{(p)}$ is the periodic Green's function defined as:

$$
G_{i j}^{(p)}\left(x_{1}\right)=\sum_{q=-\infty}^{+\infty} \hat{G}_{i j}(\gamma+q) \exp \left(j 2 \pi(q+\gamma) x_{1} / p\right)
$$

In (2), $u_{i}$ and $t_{j 2}$ are, respectively, the components of the generalized surface displacement and stress, and $u_{4}$ and $t_{42}$ are, respectively, the surface electrical potential $\phi$ and the surface charge density $Q$, i.e., the difference between the normal component of the electrical displacement in a vacuum and in the substrate. $\hat{G}_{i j}$ is the Fourier transform of the surface Green's function, i.e., the spectral Green's function. Its expression for an arbitrary piezoelectric material can be found, for instance, in [12].

As proposed by several authors [4], [20], a Chebyshev polynomial expansion of the electromechanical fields is inserted in (2) as:

$$
\begin{aligned}
t_{2 j}\left(x_{1}\right) & =\frac{1}{\sqrt{1-\bar{x}^{2}}} \sum_{m=0}^{+\infty} C_{t_{2 j}}^{(m)} T_{m}(\bar{x}), \\
Q\left(x_{1}\right) & =\frac{1}{\sqrt{1-\bar{x}^{2}}} \sum_{m=0}^{+\infty} C_{Q}^{(m)} T_{m}(\bar{x}) \\
u_{i}\left(x_{1}\right) & =\sum_{m=0}^{+\infty} C_{u_{i}}^{(m)} T_{m}(\bar{x}) \\
\phi\left(x_{1}\right) & =\sum_{m=0}^{+\infty} C_{\phi}^{(m)} T_{m}(\bar{x})
\end{aligned}
$$

where the expansion is only applied at the interface $\Gamma$ between the substrate and the electrode $\left(\left|x_{1}\right|<a / 2\right)$ and $\bar{x}=2 x_{1} / a$. In (4)-(7), the electrode is assumed to be symmetric and centered at the origin, extending along $x_{1}$ from $-a / 2$ to $a / 2$, and $T_{m}$ represents the Chebyshev polynomial of order $m .\left\{C_{f}^{(m)}\right\}$ is the vector of the Chebyshev expansion coefficients for the considered function $f$. In addition to (6) and (7), it is assumed that the stress $t_{2}$ and electrical charge $Q$ are zero in between the electrodes. By inserting (3)-(7) into (2), the following equation relating the Chebyshev expansion coefficients can be obtained for the electromechanical behavior of the substrate [4], [8]:

$$
\left\{\begin{array}{l}
C_{u_{i}}^{(m)} \\
C_{\phi}^{(m)}
\end{array}\right\}=\left[\begin{array}{ll}
A_{u t} & A_{u Q} \\
A_{\phi t} & A_{\phi Q}
\end{array}\right]\left\{\begin{array}{l}
C_{t_{2 j}}^{(n)} \\
C_{Q}^{(n)}
\end{array}\right\}
$$

Although an infinitely thin electrode can be assumed for the electrical behavior of the metal, it has been shown that the mechanical (acoustic) contribution of the strip must be taken into account [21] and is here modeled using FEM. The result of the FEM analysis, projected onto the Chebyshev polynomial expansion, can be symbolically written as [4], [8]:

$$
\begin{aligned}
& \left\{C_{u_{i}}^{(m)}\right\}= \\
& \quad \frac{1}{p}\left[\int P_{k} T_{m}\right]\left[K-\omega^{2} M\right]_{\Gamma, k l}^{-1}\left[\int P_{l} T_{n}\right]\left\{C_{t_{2 j}}^{(n)}\right\},
\end{aligned}
$$

where $\left[K-\omega^{2} M\right]_{\Gamma}$ is the usual finite element factorization matrix, where the subscript $\Gamma$ indicates that it is restricted to the electrode-substrate boundary. Matrix notation $\left[\int P_{k} T_{m}\right]$ represents the conversion of the Chebyshev expansion to a FEM polynomial interpolation $P$. The inverse of matrix $\left[K-\omega^{2} M\right]_{\Gamma, k l}$ in (9) should not be understood literally, but as representing the solution of a linear system. The mechanical contribution of the electrode then can be expressed in matrix form as:

$$
\left\{C_{u_{i}}^{(m)}\right\}=B_{u t}\left\{C_{t_{2 j}}^{(n)}\right\}
$$

The electrical boundary condition under the electrode at the origin is that the potential $\phi$ is constant and equal to $V_{0}$. From this condition the vector $C_{\phi}^{(m)}$ is easily determined. The mechanical contribution in (8) can be eliminated by inserting (10), and the resulting system solved for $C_{Q}^{(n)}$. The total electrical charge $Q_{0}$ under the electrode is computed by integration of the charge density. The result is $[19]$ :

$$
Y(\gamma)=j \frac{\pi}{2} \omega a\left(\frac{C_{Q}^{(0)}}{V_{0}}\right)
$$

\section{Approximation of FEM System Solution}

As (9) indicates, the solution of the FEM/BEM problem summarized in the preceding section requires the solution of a linear problem of the form:

$$
\left(K-\omega^{2} M\right) \mathbf{u}=\mathbf{y},
$$


where $\mathbf{y}$ represents a certain number of right-hand side vectors, for instance, one for each retained Chebyshev polynomial. Significantly, these right-hand side vectors and the matrices $K$ and $M$ are independent of the frequency. The dimension of the square matrices $K$ and $M$ is the number of degrees of freedom.

Considering the usual situation in which one is interested in computing the harmonic admittance over a given finite frequency bandwidth (e.g., in the vicinity of the stop-band), (12) has to be solved for every frequency point, which represents the main burden of the FEM/BEM method. However, observing the particular form of (12), it is seen that the only frequency dependence is explicitly in the aggregation of static matrices $K$ and $M$. This property makes possible the analytic calculation of the Taylor expansion of the solution of the linear system, as we next show. Our purpose is to obtain an approximate but fast solution of (12) limited to some frequency bandwidth.

Using the notation $\lambda=\omega^{2}$, we consider the neighborhood of the solution $\mathbf{u}_{0}$ of (12) obtained for $\lambda_{0}$, i.e., the solution of:

$$
\left(K-\lambda_{0} M\right) \mathbf{u}_{0}=\mathbf{y}
$$

Considering the small variation $\lambda=\lambda_{0}+d \lambda$ with associated solution of the linear system $\mathbf{u}=\mathbf{u}_{0}+d \mathbf{u}$, (12) reads:

$$
\left(K-\lambda_{0} M-d \lambda M\right) d \mathbf{u}=d \lambda M \mathbf{u}_{0}
$$

Note that this equation is obtained without approximation.

Let us define the matrices $A$ and $B$ by:

$$
\begin{aligned}
& A=K-\lambda_{0} M, \\
& B=A^{-1} M .
\end{aligned}
$$

The following series identity holds with a unit radius of convergence:

$$
(A-d \lambda M)^{-1}=\left(I+d \lambda B+d \lambda^{2} B^{2}+\cdots\right) A^{-1} .
$$

Injecting this identity in (14), we obtain:

$$
d \mathbf{u}=d \lambda\left(I+d \lambda B+d \lambda^{2} B^{2}+d \lambda^{3} B^{3}+\cdots\right) B \mathbf{u}_{0} .
$$

When truncated, this formula yields an approximate solution of the linear system for angular frequencies close to $\omega_{0}$, which is a special form of the general AWE method.

Although $A^{-1}$ appears explicitly in (17), it is practically not advisable to compute the inverse of matrix $A$, because this matrix is not always well conditioned. However, we next show that only a factorization of this matrix is required. Because of the positive definite character of the FEM matrices in (13), a Cholesky factorization of matrix $A$ can be performed. Alternatively, a Crout factorization also can be used. The first step is to solve (13) for the central angular frequency $\omega_{0}$, i.e., $\mathbf{u}_{0}$ is obtained where $A \mathbf{u}_{0}=\mathbf{y}$. This linear equation is easily solved using the

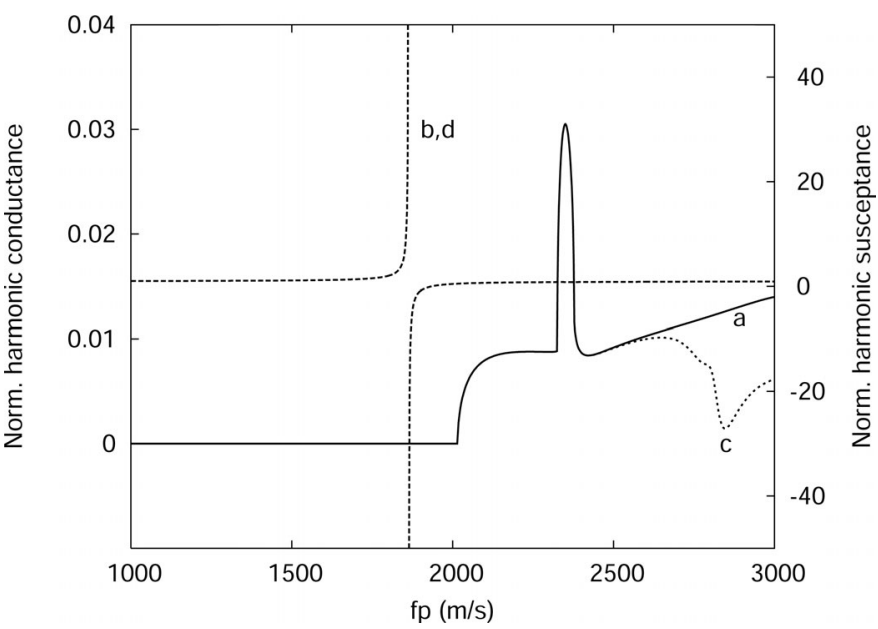

Fig. 2. Harmonic admittance for a substrate of $Y+128$ lithium niobate supporting rectangular aluminum electrodes with $h /(2 p)=0.1$ and for a metalization ratio $a / p=0.5$. The harmonic conductance (a) and harmonic susceptance (b) computed with the FEM/BEM and the harmonic conductance (c) and harmonic susceptance (d) computed with the frequency-accelerated FEM/BEM are plotted. Curves $\mathrm{b}$ and $\mathrm{d}$ cannot be distinguished visually.

factorization of matrix $A$, without having to compute the inverse of matrix $A$. The first order approximation of the solution in (18) reads:

$$
d \mathbf{u} \approx d \lambda A^{-1} M \mathbf{u}_{0}
$$

The $A^{-1} M \mathbf{u}_{0}$ term is in fact the solution of (13) with $M \mathbf{u}_{0}$ used as the right-hand side instead of $\mathbf{y}$, so that the first order approximation of the solution is readily obtained in practice. For the second order approximating term, the same procedure can be repeated, i.e., (13) must be solved with $M B \mathbf{u}_{0}$ used as the right-hand side. Iteratively, the approximating solution can be obtained to any order.

A practical iterative algorithm is the following. The approximate solution is written:

$$
\mathbf{u} \approx \mathbf{u}_{0}+d \lambda \mathbf{u}_{1}+d \lambda^{2} \mathbf{u}_{2}+\cdots
$$

$\mathbf{u}_{0}$ is first obtained as the solution of $A \mathbf{u}_{0}=\mathbf{y}$, then $\mathbf{u}_{1}$ is obtained as the solution of $A \mathbf{u}_{1}=M \mathbf{u}_{0}, \mathbf{u}_{2}$ is obtained as the solution of $A \mathbf{u}_{2}=M \mathbf{u}_{1}$, and so on.

\section{Results And Discussion}

Fig. 2 shows a comparison between the frequencyaccelerated and nonaccelerated (exact) FEM/BEM computation of the harmonic admittance of a substrate of $Y+128$ lithium niobate. The harmonic admittance is plotted as a function of the frequency period product $f p$, for rectangular aluminum electrodes with $h /(2 p)=0.1$ and for a metalization ratio $a / p=0.5$. A value $f p=2000 \mathrm{~m} / \mathrm{s}$ has been chosen as the center for the frequency-accelerated FEM/BEM computation, and seven orders are used in the approximation of (20). Seven Chebyshev polynomials for BEM, and 50 finite elements and Lagrange polynomials of 
degree 2 for FEM are used in both computations. For 1000 frequency points, a 67 -fold acceleration factor of the computation time is obtained. It can be observed that the computation results are indistinguishable over a large bandwidth extending from $f p=1000 \mathrm{~m} / \mathrm{s}$ to $f p=2500 \mathrm{~m} / \mathrm{s}$. Above $f p=2500 \mathrm{~m} / \mathrm{s}$, only the harmonic conductance differs. This gives the upper limit until which the frequencyaccelerated computation is accurate in this case.

The same computations as shown in Fig. 2 also were performed on various substrates, including $Y+42$ lithium tantalate and $Y+36-Z$ quartz, and for several values of the parameter $h /(2 p)$. The same features as described above were observed in all cases. The number of orders retained in the Taylor expansion of (20) was varied from 3 to 20 to verify whether the range of validity of the approximation could be extended. This range of validity was found to be only slightly dependent on the order of the approximation, and it could not be extended significantly over the seven orders approximation used for plotting Fig. 2. It also was verified that the approximation is correct when the central frequency is shifted. For instance, with a central value $f p=3000 \mathrm{~m} / \mathrm{s}$, the frequencyaccelerated FEM/BEM computation closely approximates the exact solution from $f p=2000 \mathrm{~m} / \mathrm{s}$ to $f p=3500 \mathrm{~m} / \mathrm{s}$.

The range of validity of the approximation of (20) is certainly limited to frequencies for which a Taylor expansion can be considered for the acoustic fields inside the electrodes. This is certainly not the case when some mode of the electrode is excited because a resonant behavior is then expected. Conversely, for all frequencies sufficiently far from a mechanical resonance inside the electrodes, or if the resonance is damped out by losses (for instance, due to radiation inside the substrate), the approximation is expected to be valid. This has been the situation with all piezoelectric cuts and waves considered during this work, and no resonances in the electrodes were observed. However, when considering a metal different from aluminum for the electrodes or fast surface acoustic waves (e.g., longitudinal leaky SAWs), care should be taken that the accelerated results are meaningful. Although this is not done in this work, it should be noted that AWE methods can take care of poles, i.e., resonances. This would complicate and probably slow down the method presented in this paper.

\section{Conclusions}

The FEM/BEM computation model applied to SAW devices requires the solution of a large FEM linear system for each frequency point. A Taylor expansion of the solution of this system has been proposed to obtain an approximate solution that is valid on a large frequency bandwidth. The Taylor expansion orders are easily and efficiently computed with a simple recursion formula. The approximation method belongs to the class of asymptotic evaluation waveform methods. The approximate solution has been shown to be very accurate, provided resonance modes of the electrodes are not excited, and it vastly re- duces the computation time. The AWE models could possibly benefit other ultrasonics applications that are modeled in the spectral domain using FEM, as exemplified by $[22]$.

\section{ACKNOWLEDGMENTS}

The authors are grateful to Pascal Ventura, William Steichen, Thomas Pastureaud, Raphaël Lardat, and Marc Solal for enlightening discussions.

\section{ReFERENCES}

[1] H. P. Reichinger and A. R. Baghai-Wadji, "Dynamic 2D analysis of SAW devices including mass-loading," in Proc. IEEE Ultrason. Symp., 1992, pp. 263-266.

[2] H. P. Reichinger, A. R. Baghai-Wadji, and F. J. Seifert, "Stress pattern on the electrode/substrate interfaces in SAWdevices," in Proc. IEEE Ultrason. Symp., 1993, pp. 153-156.

[3] P. Ventura, J. Desbois, and L. Boyer, "A mixed FEM/analytical model of the electrode mechanical perturbation for SAW and PSAW propagation," in Proc. IEEE Ultrason. Symp., 1993, pp. 205-208.

[4] P. Ventura, J. M. Hodé, and M. Solal, "A new efficient combined FEM and periodic Green's function formalism for the analysis of periodic SAW structures," in Proc. IEEE Ultrason. Symp., 1995, pp. 263-269.

[5] G. Endoh, K. Hashimoto, and M. Yamaguchi, "Surface acoustic wave propagation characterization by finite element method and spectral domain analysis," Jpn. J. Appl. Phys., vol. 34, pp. 26382641, 1995.

[6] P. Ventura, J.-M. Hodé, M. Solal, J. Desbois, and J. Ribbe, "Numerical methods for SAW propagation characterization," in Proc. IEEE Ultrason. Symp., 1998, pp. 175-186.

[7] J. Koskela, V. P. Plessky, and M. M. Salomaa, "SAW/LSAW COM parameter extraction from computer experiments with harmonic admittance of a periodic array of electrodes," IEEE Trans. Ultrason., Ferroelect., Freq. Contr., vol. 46, pp. 806-816, 1999.

[8] P. Ventura, J.-M. Hodé, J. Desbois, and M. Solal, "Combined FEM and Green's function analysis of periodic SAW structure, application to the calculation of reflection and scattering parameters," IEEE Trans. Ultrason., Ferroelect., Freq. Contr., vol. 48, pp. 1259-1274, 2001.

[9] V. Laude, A. Khelif, T. Pastureaud, and S. Ballandras, "Generally polarized acoustic waves trapped by high aspect ratio electrode gratings on piezoelectric substrates," J. Appl. Phys., vol. 90, pp. 2492-2497, 2001.

[10] K. Hashimoto, G. Endoh, M. Ohmaru, and M. Yamaguchi, "Analysis of SAWs obliquely propagating under metallic gratings with finite thickness," Jpn. J. Appl. Phys., vol. 35, pp. 30063009, 1996.

[11] V. Laude and S. Ballandras, "Slowness curves and characteristics of surface acoustic waves propagating obliquely in periodic finitethickness electrode gratings," J. Appl. Phys., vol. 94, pp. 1235$1242,2003$.

[12] Y. Zhang, J. Desbois, and L. Boyer, "Characteristic parameters of surface acoustic waves in a periodic metal grating on a piezoelectric substrate," IEEE Trans. Ultrason., Ferroelect., Freq. Contr., vol. 40, pp. 183-192, 1993.

[13] K. Blötekjær, K. A. Ingebrigtsen, and H. Skeie, "A method for analysing waves in structures consisting of metal strips on dispersive media," IEEE Trans. Electron. Devices, vol. ED-20, pp. 1133-1138, 1973.

[14] K. Blötekjær, K. A. Ingebrigtsen, and H. Skeie, "Acoustic surface waves with periodic metal strips on the surface," IEEE Trans. Electron. Devices, vol. ED-20, pp. 1139-1146, 1973.

[15] L. T. Pillage and R. A. Rohrer, "Asymptotic waveform evaluation for timing analysis," IEEE Trans. Computer-Aided Design, vol. 9, pp. 352-366, 1990. 
[16] J. E. Bracken, V. Raghavan, and R. A. Rohrer, "Interconnect simulation with asymptotic waveform evaluation," IEEE Trans. Circuits Syst. I, vol. 39, pp. 869-878, 1992.

[17] T. K. Tang and M. S. Nakhla, "Analysis of high-speed VLSI interconnects using the asymptotic waveform evaluation technique," IEEE Trans. Computer-Aided Design, vol. 11, pp. 341352, 1992.

[18] E. Chiprout and M. S. Nakhla, Asymptotic Waveform Evaluation and Moment Matching for Interconnect Analysis. Norwell, MA: Kluwer, 1994.

[19] Y. Fusero, S. Ballandras, J. Desbois, J.-M. Hodé, and P. Ventura, "SSBW to PSAW conversion in SAW devices using heavy mechanical loading," IEEE Trans. Ultrason., Ferroelect., Freq. Contr., vol. 49, pp. 805-814, 2001.

[20] V. P. Plessky and T. Thorvaldsson, "Rayleigh waves and leaky SAWs in periodic systems of electrodes: Periodic Green functions analysis," Electron. Lett., vol. 28, pp. 1317-1319, 1992.

[21] A. R. Baghai-Wadji and A. A. Maradudin, "Shear horizontal surface acoustic waves on large amplitude gratings," Appl. Phys. Lett., vol. 59, pp. 1841-1843, 1991.

[22] C. H. Lee, H. W. Joo, J. S. Rho, H. S. Cho, and H. K. Jung, "Inversion of piezoelectric material coefficients by using finite element method with asymptotic waveform evaluation," in Proc. IEEE Ultrason. Symp., 2002, pp. 643-646.

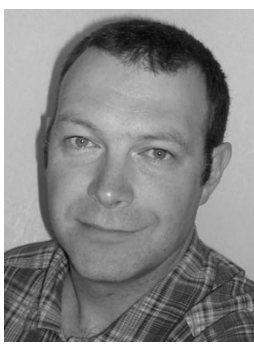

Vincent Laude (M'00) was born in Bourgla-Reine, France, in 1968. He received an Engineering Diploma in 1990 from the Ecole Supérieure d'Optique, and a Ph.D. degree in physics in 1994 from Paris XI University, both in Orsay, France. He received his Habilitation à Diriger des Recherches degree from the Université de Franche-Comté in 2002.

From 1995 to 1999 , he was a researcher at Thomson-CSF Corporate Research Laboratory (now Thales TRT) in Orsay, France, where he worked on various aspects of optical signal processing, wavefront sensing, and ultrashort laser pulses. In 2000 he joined Thomson-CSF Microsonics in Sophia-Antipolis, France, to work on surface acoustic wave propagation. At the end of that year, he joined the Laboratoire de Physique et Métrologie des Oscillateurs, Centre National de la Recherche Scientifique in Besançon, France.

He currently is interested in the propagation of surface, interface, and guided acoustic waves, their interaction with micro-structures, and phononic band gap materials. He is a member of IEEE/UFFC.

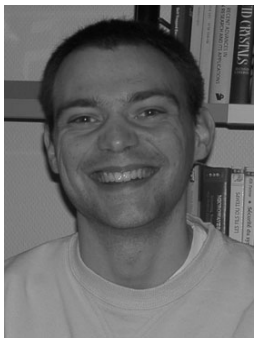

Alexandre Reinhardt was born in 1977 in Pavillons-sous-Bois, France. He received the engineering degree from the Ecole Centrale de Lille, Lille, France, (formerly Institut Industriel du Nord) in 2001. The same year, he received the DEA (master) degree in electronics applied to ultrasound and imagery from the University of Valenciennes, Valenciennes, France. In 2001, he joined the Laboratoire de Physique et Métrologie des Oscillateurs, Besançon, France, as a Ph.D. student. He is also with Temex Microsonics, Sophia Antipo-

lis, France.

His current interests include the simulation of waves in stratified structures and film bulk acoustic resonator devices.

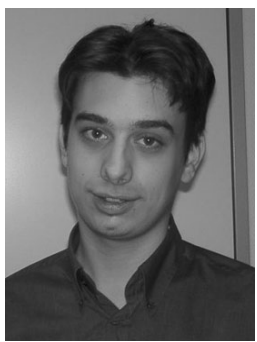

Mikaël Wilm was born in Athis-Mons, France, in 1977. He received an Engineering Diploma in 2000 from the Ecole Nationale Supérieure de Mécanique et de Microtechniques in Besançon, France, and a DEA degree in 2001 from the Université de FrancheComté, Besançon, France. Then he joined the Laboratoire de Physique et Métrologie des Oscillateurs, Centre National de la Recherche Scientifique in Besançon, France, as a Ph.D. student.

He currently is interested in acoustic periodic devices, particularly in periodic piezoelectric composite structures for medical imaging and nondestructive evaluation.

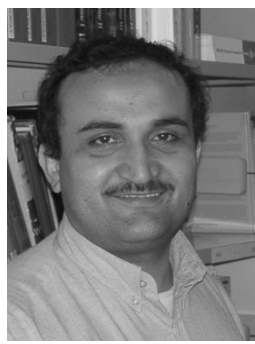

Abdelkrim Khelif was born in 1971 in Bouira, Algeria. He received the DEA degree and the Ph.D. degree in materials science from the Université de Lille I, Lille, France. His dissertation was dedicated to the theory of vibrations of and acoustic scattering by supported wires on a surface.

In 2000, he joined the Laboratoire de Physique des Solides of the University of Namur, Belgium, as a postdoc researcher. In 2002, he joined the Laboratoire de Physique et Métrologie des Oscillateurs, Centre National de la Recherche Scientifique in Besançon, France.

He currently is interested in the propagation of acoustic waves in elastic band gap materials.

Sylvain Ballandras was born in 1965 in Strasbourg, France. He received the DEA degree in acousto-opto-electronique et vibrations and the Ph.D. degree in science pour l'Ingénieur from the Université de Franche-Comté, Besançon, France.

He joined the Laboratoire de Physique et Métrologie des Oscillateurs, Centre National de la Recherche Scientifique (LPMO/CNRS) in Besançon, France, as a permanent staff member in 1991. He has been working in the fields of SAW sensitivity to physical perturbations and in parallel in microtechnologies such as LIGA and stereolithography. He received the Habilitation à Diriger des Recherches degree from the Université de Franche-Comté in 1997. He joined Thomson Microsonics, Sophia Antipolis, France, for 1 year as a research engineer. He returned to the LPMO in 1998. Since 1999, he has been heading the acoustics and microsonics research group at the LPMO.

His current interests are in the application of SAW devices (sensors), in the development of numerical models, and new technologies for ultrasound transducers devoted to acoustic imaging and nondestructive control. 